\title{
Cohesive properties of group-III nitrides: A comparative study of all-electron and pseudopotential calculations using the generalized gradient approximation
}

\author{
M. Fuchs, J. L. F. Da Silva, C. Stampfl,* J. Neugebauer, and M. Scheffler \\ Fritz-Haber-Institut der Max-Planck-Gesellschaft, Faradayweg 4-6, 14195 Berlin-Dahlem, Germany
}

(Received 23 January 2002; published 24 June 2002)

\begin{abstract}
We compare the performance of generalized gradient approximations (GGA's) and the local-density approximation (LDA) in density-functional calculations of the cohesive properties of cubic AlN, GaN, and InN. Employing the widely adopted pseudopotential approach, the calculated data are found to depend significantly on the treatment of the core states of the group-III ions, hampering a conclusive assessment of the GGA and LDA. Here we perform all-electron full-potential linearized-augmented plane-wave calculation, which we use to (i) scrutinize the results of pseudopotential calculations, and (ii) provide a proper distinction between the GGA and LDA functionals. We show that the accuracy of pseudopotential calculations is comparable to that of all-electron calculations only if the $\mathrm{Ga}$ and In semicore $d$ states are treated as valence rather than core states. We also show that the use of an $f$-like local component can further improve the transferability of the $\mathrm{Ga}$ and In pseudopotentials. Regarding the PBE- (Perdew-Burke-Enzerhof-) GGA [Phys. Rev. Lett. 77, 3865 (1996)] we find that the cohesive energies of the group-III nitride crystals (and those of the elemental metals) agree closely with experimental data whereas they are overestimated within the LDA. Lattice parameters are described with similar accuracy within the PBE-GGA and LDA. On the other hand we find that the heats of formation of the group-III nitrides are underestimated by the PBE-GGA and given more accurately by the LDA. For the PBE-GGA, the underestimate is mainly due to the fact that it still overestimates the bond strength of the $\mathrm{N}_{2}$ molecule. For the LDA, the heat of formation turns out only slightly too large, because of a fortuitous cancellation of the (larger) errors in the $\mathrm{N}_{2}$ molecule and the bulk crystals. Several other GGA functionals are able to improve over the PBE-GGA for molecules like $\mathrm{N}_{2}$ due to stronger gradient corrections. Here we find that such more nonlocal GGA's significantly underestimate the cohesive energies of the group-III nitride (and metal) crystals and even further underestimate their heats of formation.
\end{abstract}

DOI: 10.1103/PhysRevB.65.245212

PACS number(s): 71.55.Eq, 71.55.Ak, 71.15.Mb

\section{INTRODUCTION}

Their wide direct band gaps and large thermal and chemical stability make the group-III nitrides ( $\mathrm{AlN}, \mathrm{GaN}, \mathrm{InN}$, and their alloys) useful materials for optoelectronic devices that work in the visible to UV range of the optical spectrum. ${ }^{1}$ The comprehensive control of device characteristics of these materials remains a technological challenge and still calls for a detailed understanding of their physical and chemical behavior. This task is also being pursued in theoretical studies, with recent work addressing, e.g., structural, dynamical, ${ }^{2,3}$ and optical properties, ${ }^{4}$ defects ${ }^{5,6}$ and dopants, ${ }^{7-9}$ interface $^{10}$ and surface properties. ${ }^{11,12}$ Such studies often build on totalenergy calculations within density-functional theory, where, routinely, many-electron exchange correlation is described in the local-density approximation (LDA). ${ }^{13}$ Yet one is well aware that use of the LDA leads to overestimated bond strengths in molecules and solids, ${ }^{14}$ a shortcoming seen for the group-III nitrides too. A more accurate account of exchange correlation is thus desirable, particularly for scenarios where bonds are broken or formed anew, say, when studying bulk impurities or the behavior of reactants during crystal growth.

In this paper we examine whether generalized gradient approximations $^{15,16}$ (GGA's) are able to improve over the LDA for the group-III nitrides. Indeed, for many molecules $^{17,18}$ and solids ${ }^{19-22}$ GGA's have been shown to yield more accurate binding energies than the LDA. Reaction and activation energies for various chemical reactions ${ }^{23,24}$ as well as for the adsorption of adparticles on surfaces of metals $^{25}$ and semiconductors ${ }^{26}$ are likewise improved. While GGA's tend to enlarge crystal equilibrium volumes, they do not, in general, lead to more accurate stuctural or elastic parameters than the LDA. For instance, in many covalent semiconductors the lattice parameters turn out too large to a comparable degree as they turn out too small in the LDA. ${ }^{27,28}$

In this study we carry out all-electron full-potential linearized-augmented plane-wave (FP-LAPW) calculations to determine the structural and cohesive properties of cubic $\mathrm{AlN}, \mathrm{GaN}, \mathrm{InN}$, and their constituents (Al, $\mathrm{Ga}, \mathrm{In}$, and $\mathrm{N}_{2}$ ) within the LDA (Ref. 29) and the PBE- (Perdew-BurkeEnzerhof-) GGA. ${ }^{30}$ Such a comparison has been attempted in an all-electron linear muffin-tin orbital (LMTO) framework before but restricted to structural and elastic properties. ${ }^{31}$ While several pseudopotential studies have been performed, their results differ significantly with regard to the quantitative effect of the GGA in these materials: Using the PW91(Perdew-Wang 1991) GGA (Ref. 15), Stampfl and Van de Walle $^{32}$ found the lattice parameters of $\mathrm{GaN}$ and $\mathrm{InN}$ overestimated and their cohesive energies underestimated compared to experimental data. Also, $\mathrm{InN}$ is found endothermic with a positive heat of formation. Using the PBE-GGA Zoroddu et al. ${ }^{33}$ found lattice parameters less overestimated and cohesive energies in distinctly better agreement with experiment than in Ref. 32. On the other hand, Miotto et al. ${ }^{34}$ found the PBE-GGA lattice parameter of $\mathrm{GaN}$ underestimated with respect to experiment, and in fact almost un- 
changed compared to the LDA value. The different results for the PW91-GGA and the PBE-GGA are surprising, as for many other solids both functionals perform similarly. ${ }^{22}$ Two interpretations are conceivable though: First, that different GGA's indeed perform distinctly for the group-III nitrides, which are rather ionic materials with short and strong bonds. We point out that on such systems GGA's have been tested less $^{30,31}$ than on metals and covalent semiconductors. Learning about the performance of the GGA for the group-III nitrides is thus of interest regarding both the understanding and further refinements of current exchange-correlation functionals. Second, that the differences in the results of the different pseudopotential calculations are a consequence of the use of different levels of approximation in the pseudopotential approach rather than the use of different exchange-correlation functionals. In particular, it has been argued before $e^{5,35,36}$ that the Ga $3 d$ semicore electrons, rather unusual for III-V semiconductors, act as valence electrons and are essential to obtain accurate structural properties. Indeed Stampfl et al. and Zoroddu et al. treated the Ga $3 d$ and In $4 d$ states as valence states, whereas Miotto et al. treated them as core states, obtaining markedly different lattice parameters. On the other hand, Stampfl et al. used norm-conserving pseudopotentials (where the $s$-like component was chosen as the local potential), whereas Zoroddu et al. used the ultrasoft pseudopotential approach, obtaining markedly different cohesive energies. Given the practical importance of the pseudopotential approach, it is desirable to identify and avoid such pseudopotential related uncertainties. Using our all-electron results as a reference we discuss the accuracy or transferability of (norm-conserving) pseudopotentials as they are typically used for group-III nitrides. We thereto explicitly compare $\mathrm{Ga}$ and In pseudopotentials that treat the semicore $d$ states either as core or as valence states. Our results show that pseudopotentials must treat the Ga $3 d$ or In $4 d$-states as valence states in order to achieve an accuracy comparable to that of the all-electron calculations, regardless of whether the LDA or the PBE-GGA is used. We also find that the calculated properties of $\mathrm{GaN}$ (or InN) are significantly affected by the treatment of the local part of the cation pseudopotentials.

Regarding the GGA's, our calculations demonstrate both favorable and unfavorable aspects for the group-III nitride systems. On the one hand, within the PBE-GGA, the cohesive energies agree closely with experimental values. As for other bulk semiconductors the PBE-GGA thus corrects the overbinding found for the LDA. At the same time both functionals yield the lattice parameters with similar accuracy, slightly underestimated with the LDA and slightly overestimated within the PBE-GGA. On the other hand, the heat of formation of the group-III nitride crystals, given through the reaction

$$
X \mathrm{~N}(\text { solid }) \rightleftharpoons X(\text { solid })+\frac{1}{2} \mathrm{~N}_{2}(\text { gas })
$$

with $X$ for $\mathrm{Al}, \mathrm{Ga}$, or In,

for the decomposition of the bulk nitrides into the elemental metal and nitrogen phases, is markedly underestimated by the PBE-GGA. In particular, $\mathrm{InN}$ is described as endothermic (unstable), as was found also for the PW91-GGA. ${ }^{32}$ This apparent shortcoming can be understood to be (mainly) due to the fact that the PBE-GGA significantly overestimates the binding energy of the free $\mathrm{N}_{2}$ molecules, whereas it yields reasonable cohesive energies of the bulk crystals: the net effect is an underestimate of the heat of formation. The LDA yields more accurate heats of formation, but it is essentially due to a cancellation of its larger errors for the binding energy of the $\mathrm{N}_{2}$ molecule and the cohesive energy of the bulk crystals. Interestingly we find similar limitations when we employ instead of the PBE-GGA the recent "revised" PBEGGA functionals of Zhang and Yang ${ }^{37}$ (revPBE-GGA) and Hammer et al. ${ }^{38}$ (RPBE-GGA), or the earlier BLYP- (BeckeLee-Yang-Parr) GGA. ${ }^{39,40}$ Our motivation for the use of these alternative GGA functionals is that they can lead to improved molecular binding energies and do so for the $\mathrm{N}_{2}$ dimer, where the PBE-GGA is less accurate than for the group-III nitride or metal bulk crystals. However, in contrast to the PBE-GGA, the revPBE, RPBE, and BLYP-GGA's seriously underestimate the cohesive energy of the bulk crystals and, in the end, underestimate the heat of formation for the group-III nitrides even more than the PBE-GGA. Inspecting the density dependence of the different GGA functionals we discuss our findings as a consequence of the increasingly nonlocal character of the gradient corrections when going from the PBE-GGA to the alternative revPBE, RPBE, and BLYP-GGA's.

We expect that our calculations for the cubic structures are also representative for the wurtzite ground-state structures of the group-III nitrides, since the energy differences between both phases are estimated as $\$ 45 \mathrm{meV} /$ pair. $^{32,41}$ While not discussed further in this paper, we note that the above GGA's and the LDA produce very similar Kohn-Sham bands in the group-III nitrides, except for small deformation potential effects related to differently predicted atomic structures. ${ }^{32}$ In particular, the Kohn-Sham band gaps, when interpreted as electronic excitation energies, are clearly too small, analogous to what is found (and understood) in other semiconductors. ${ }^{31}$ As such this is not troublesome in the present study of perfect crystals, but may require care when gap states play a role as in surface or defect systems. ${ }^{42}$

This paper is organized as follows. In Sec. II we outline the computational aspects of our all-electron and pseudopotential calculations, and examine the pseudopotential related approximations. In Sec. III we discuss our LDA and PBEGGA results for the bulk nitrides' and their constituents' binding properties, and then compare the different GGA functionals. Section IV contains our conclusions.

\section{CALCULATIONAL PROCEDURE}

We perform density-functional total energy calculations using both the FP-LAPW method and the pseudopotential plane-wave method. Below we describe the computation of the lattice parameters and binding energies, which is entirely analogous in both methods, and then turn to the more specific aspects of either approach. For the bulk nitride and $\mathrm{Al}$ crystals we find the equilibrium unit-cell volumes or lattice 
TABLE I. Comparison of results from pseudopotential and all-electron FP-LAPW calculations for the lattice constant $a$, cohesive energy $E_{b}$, and the enthalpy of formation $\Delta H_{\mathrm{f}}$ of cubic group-III nitrides. Different pseudopotentials are used as described in Sec. II C and indicated in the second column. Given in brackets are the deviations of the pseudopotential from the respective FP-LAPW results. Results refer to the LDA and PBE-GGA functionals.

\begin{tabular}{|c|c|c|c|c|c|c|c|c|c|c|c|c|c|}
\hline \multirow[b]{3}{*}{ AlN } & \multirow{3}{*}{$\frac{\text { Method }}{\mathrm{Al}^{3+}}$} & \multicolumn{4}{|c|}{$a(\AA)$} & \multicolumn{4}{|c|}{$E_{b}(\mathrm{eV})$} & \multicolumn{4}{|c|}{$\Delta H_{\mathrm{f}}(\mathrm{eV})$} \\
\hline & & \multicolumn{2}{|c|}{ LDA } & \multicolumn{2}{|r|}{ PBE } & \multicolumn{2}{|c|}{ LDA } & \multicolumn{2}{|c|}{ PBE } & \multicolumn{2}{|c|}{ LDA } & \multicolumn{2}{|c|}{ PBE } \\
\hline & & 4.30 & $(-0.04)$ & 4.38 & $(-0.02)$ & 13.39 & $(+0.14)$ & 11.55 & $(+0.01)$ & -3.42 & $(-0.03)$ & -2.67 & $(-0.03)$ \\
\hline & $\mathrm{Al}^{3+}$ and NLCV XC & 4.33 & $(-0.01)$ & 4.40 & $(0.00)$ & 13.35 & $(+0.10)$ & 11.58 & $(+0.06)$ & -3.38 & $(+0.01)$ & -2.70 & $(+0.00)$ \\
\hline & FP-LAPW & \multicolumn{2}{|c|}{4.34} & \multicolumn{2}{|r|}{4.40} & \multicolumn{2}{|c|}{13.25} & \multicolumn{2}{|c|}{11.52} & \multicolumn{2}{|c|}{-3.39} & \multicolumn{2}{|c|}{-2.70} \\
\hline & Experiment $^{\mathrm{a}}$ & \multicolumn{4}{|c|}{4.38} & \multicolumn{4}{|c|}{11.54} & \multicolumn{4}{|c|}{-3.25} \\
\hline \multirow[t]{6}{*}{$\mathrm{GaN}$} & $\mathrm{Ga}^{3+}$ & 4.30 & $(-0.16)$ & 4.50 & $(-0.05)$ & 12.39 & $(+1.59)$ & 9.37 & $(+0.51)$ & -2.76 & $(-1.20)$ & -1.19 & $(-0.28)$ \\
\hline & $\mathrm{Ga}^{3+}$ and NLCV XC & 4.43 & $(-0.03)$ & 4.51 & $(-0.04)$ & 11.05 & $(+0.25)$ & 9.21 & $(+0.25)$ & -1.83 & $(-0.27)$ & -1.11 & $(-0.20)$ \\
\hline & $\mathrm{Ga}^{13+}$ local $s$ & 4.51 & $(+0.05)$ & 4.60 & $(+0.05)$ & 10.42 & $(-0.38)$ & 8.53 & $(-0.32)$ & -1.19 & $(+0.37)$ & -0.55 & $(+0.36)$ \\
\hline & $\mathrm{Ga}^{13+}$ local $f$ & 4.47 & $(+0.01)$ & 4.57 & $(+0.02)$ & 10.68 & $(-0.12)$ & 8.74 & $(-0.12)$ & -1.38 & $(+0.18)$ & -0.71 & $(+0.20)$ \\
\hline & FP-LAPW & & 4.46 & & 4.55 & & .80 & & 3.86 & & .56 & & .91 \\
\hline & Experiment $^{\mathrm{a}}$ & \multicolumn{4}{|c|}{4.52} & \multicolumn{4}{|c|}{8.96} & \multicolumn{4}{|c|}{-1.27} \\
\hline \multirow[t]{6}{*}{ InN } & $\mathrm{In}^{3+}$ & 4.68 & $(-0.26)$ & 4.91 & $(-0.14)$ & 11.68 & $(+2.49)$ & 8.53 & $(+1.18)$ & -2.31 & $(-1.98)$ & -0.57 & $(-0.81)$ \\
\hline & $\mathrm{In}^{3+}$ and NLCV XC & 4.89 & $(-0.05)$ & 4.99 & $(-0.06)$ & 9.77 & $(+0.58)$ & 7.53 & $(+0.18)$ & -0.78 & $(-0.45)$ & -0.31 & $(-0.55)$ \\
\hline & $\operatorname{In}^{13+}$ local $s$ & 4.99 & $(+0.05)$ & 5.11 & $(+0.06)$ & 8.87 & $(-0.32)$ & 7.10 & $(-0.25)$ & 0.02 & $(+0.35)$ & 0.55 & $(+0.31)$ \\
\hline & $\operatorname{In}^{13+}$ local $f$ & 4.95 & $(+0.01)$ & 5.06 & $(+0.01)$ & 9.11 & $(-0.08)$ & 7.31 & $(-0.04)$ & -0.19 & $(+0.14)$ & 0.35 & $(+0.11)$ \\
\hline & FP-LAPW & & 4.94 & & 5.05 & & 19 & & 7.35 & & .33 & & 24 \\
\hline & Experiment ${ }^{\mathrm{a}}$ & \multicolumn{4}{|c|}{4.98} & \multicolumn{4}{|c|}{7.72} & \multicolumn{4}{|c|}{-0.32} \\
\hline
\end{tabular}

${ }^{\mathrm{a}}$ See Tables II-X.

parameters by minimizing the total energy as a function of the cell volume. We compute the total energies for a set of volumes over a range of about $\pm 15 \%$ around the experimental value. Fitting these to Murnaghan's equation of state we obtain the values for the equilibrium cell volume, bulk modulus, and total energy. ${ }^{43}$ In the case of $\alpha$-Ga, we minimize the total energy for a fixed volume with respect to the positions of the basis atoms, using the atomic forces, and with respect to the shape parameters of the orthorhombic unit cell, using polynomial interpolation. For In we do a similar minimization with respect to the $c / a$ ratio of the centered tetragonal unit cell. For the case of the $\mathrm{N}_{2}$ molecule we determine the equilibrium bond parameters in an analogous way. In computing the total energies of the free atoms and the $\mathrm{N}_{2}$ dimer we use a cubic supercell with $b=15$ bohr side length and work with the single $\mathbf{k}$ point $(1 / 4,1 / 4,1 / 4)$ $\times(2 \pi / b)$. To carry out the Brillouin-zone summations we use a $6 \times 6 \times 6$ mesh of special $\mathbf{k}$ points ${ }^{44}$ ( 28 points in the irreducible wedge of the Brillouin zone). For the bulk metals we use similar meshes with $72(\mathrm{Al}), 18(\mathrm{Ga})$, and 400 (In) points, and employ a Fermi broadening corresponding to an electronic temperature of up to $T^{\mathrm{el}}=0.15 \mathrm{eV}^{45}$ From careful convergence tests of our LAPW and plane-wave basis sets we estimate that the total energies and hence the binding energies are converged to better than $30 \mathrm{meV} /$ atom.

The cohesive energies of the group-III nitrides (Tables I-IV) are calculated from the ground-state total energies of the crystals $E_{\text {tot }}^{\mathrm{XN}(\mathrm{c})}$ and the free atoms $E_{\text {tot }}^{\mathrm{X}(\mathrm{at}), \mathrm{N}(\mathrm{at})}$ as

$$
E_{b}^{X \mathrm{~N}}=-E_{\mathrm{tot}}^{X \mathrm{~N}(\mathrm{c})}+E_{\mathrm{tot}}^{X(\mathrm{at})}+E_{\mathrm{tot}}^{\mathrm{N}(\mathrm{at})},
$$

where $X$ stands for $\mathrm{Al}, \mathrm{Ga}$, or In. The cohesive energies of the elemental metals (Tables V-VIII) and the binding energy of the $\mathrm{N}_{2}$ dimer (Table IX) are likewise obtained. The heat of formation, $\Delta H_{\mathrm{f}}^{X \mathrm{~N}}$, (Tables I and $\mathrm{X}$ ) is obtained as

$$
\Delta H_{\mathrm{f}}^{\mathrm{XN}}=-\left(E_{\mathrm{tot}}^{X(\mathrm{c})}+E_{\mathrm{tot}}^{\mathrm{N}_{2}}\right)+E_{\mathrm{tot}}^{X \mathrm{~N}(\mathrm{c})} \equiv E_{b}^{X}+E_{b}^{\mathrm{N}_{2}}-E_{b}^{X \mathrm{~N}} .
$$

For $\Delta H_{\mathrm{f}}^{X \mathrm{~N}}<0$, the nitride crystal $X \mathrm{~N}$ is thermodynamically stable.

The total energies of the free atoms includes the spinpolarization energies, which we evaluate on the all-electron level with spherically symmetric electron ground-state densities. For the open-shell atoms $\mathrm{Al}, \mathrm{Ga}$, and $\mathrm{In}$, the

TABLE II. Cohesive properties of cubic AlN from pseudopotential (PP) and FP-LAPW all-electron calculations. The first column indicates the exchange-correlation functional employed. Shown are the lattice constant $a$, the bulk modulus $B$, and the cohesive energy $E_{b}$. The latter includes the spin corrections for the the free $\mathrm{Al}$ and

\begin{tabular}{|c|c|c|c|c|}
\hline $\mathrm{XC}$ & Method & $a(\AA)$ & $B(\mathrm{GPa})$ & $E_{b}(\mathrm{eV})$ \\
\hline LDA & $\mathrm{PP}$ & 4.33 & 201 & 13.35 \\
\hline LDA & LAPW & 4.34 & 209 & 13.25 \\
\hline LDA & LAPW $^{\mathrm{a}}$ & 4.342 & 207 & \\
\hline LDA & $\mathrm{LMTO}^{\mathrm{b}}$ & 4.345 & 207 & \\
\hline PBE & $\mathrm{PP}$ & 4.40 & 191 & 11.58 \\
\hline PBE & LAPW & 4.40 & 191 & 11.54 \\
\hline PBE & $\mathrm{LMTO}^{\mathrm{b}}$ & 4.40 & & \\
\hline \multicolumn{2}{|c|}{ Experiment ${ }^{\mathrm{c}}$} & 4.38 & 202 & 11.52 \\
\hline
\end{tabular}
$\mathrm{N}$ atom (see Tables $\mathrm{V}$ and IX).

${ }^{\mathrm{a}}$ Reference 36.

${ }^{\mathrm{b}}$ Reference 31 .

${ }^{\mathrm{c}}$ Taken from Refs. $62(a), 79(B)$, and $80\left(E_{b}\right)$. 
TABLE III. Cohesive properties of cubic GaN. As for Table II.

\begin{tabular}{|c|c|c|c|c|}
\hline $\mathrm{XC}$ & Method & $a(\AA)$ & $B(\mathrm{GPa})$ & $E_{b}(\mathrm{eV})$ \\
\hline LDA & $\mathrm{PP}$ & 4.47 & 198 & 10.68 \\
\hline LDA & LAPW & 4.46 & 200 & 10.80 \\
\hline LDA & LAPW $^{a}$ & 4.460 & 187 & \\
\hline LDA & LMTO $^{b}$ & 4.464 & & \\
\hline PBE & PP & 4.57 & 169 & 8.74 \\
\hline PBE & LAPW & 4.55 & 172 & 8.86 \\
\hline PBE & LMTO $^{b}$ & 4.57 & & \\
\hline \multicolumn{2}{|c|}{ Experiment ${ }^{c}$} & 4.52 & 190 & 8.96 \\
\hline
\end{tabular}

${ }^{\mathrm{a}}$ Reference 36.

${ }^{\mathrm{b}}$ Reference 31 .

${ }^{\mathrm{c}}$ Taken from Refs. $62(a), 79(B)$, and $80\left(E_{b}\right)$.

GGA's can lead to slightly lower ground-state energies, by less than $0.1 \mathrm{eV},{ }^{46}$ when nonspherical ground-state densities are allowed for. We have not included these small corrections when evaluating the cohesive energies. Like the atomic spinpolarization energies they are not relevant for calculating the heat of formation.

\section{A. All-electron FP-LAPW calculations}

In the FP-LAPW method (see, e.g., Ref. 47) both core and valence states are treated fully self-consistently, in particular, no shape approximations are made for the effective potential and the core states are allowed to relax with the atomic environment. We use the WIEN97 implementation, ${ }^{48}$ which allows to include local orbitals in the basis and thus enables the consistent treatment of semicore and valence states in one energy window. ${ }^{47}$ Our calculations treat the core states relativistically and the valence states in a scalar relativistic approximation. We mention that an entirely nonrelativistic treatment increases the cohesive energies by $0.01(\mathrm{AlN})$, $0.50(\mathrm{GaN})$, and $0.80 \mathrm{eV} /$ pair $(\mathrm{InN})$, and also leads to somewhat larger lattice constants, following the same pattern as noted for II-VI compounds. ${ }^{49}$ A high quality basis set is employed throughout, where we choose the radii of the muffintin spheres $R$ and the plane-wave cutoff sphere $K_{\max }$ such that $R K_{\max } \geqslant 9$ for all systems. In addition, we include local

TABLE IV. Cohesive properties of cubic InN. As for Table II.

\begin{tabular}{|c|c|c|c|c|}
\hline $\mathrm{XC}$ & Method & $a(\AA)$ & $B(\mathrm{GPa})$ & $E_{b}(\mathrm{eV})$ \\
\hline LDA & PP & 4.95 & 145 & 9.11 \\
\hline LDA & LAPW & 4.94 & 145 & 9.19 \\
\hline LDA & LAPW $^{\mathrm{a}}$ & 4.932 & 140 & \\
\hline LDA & LMTO $^{\text {b }}$ & 4.957 & & \\
\hline PBE & PP & 5.06 & 120 & 7.31 \\
\hline PBE & LAPW & 5.05 & 122 & 7.35 \\
\hline PBE & LMTO $^{\text {b }}$ & 5.06 & & \\
\hline \multicolumn{2}{|c|}{ Experiment ${ }^{\mathrm{c}}$} & 4.98 & 137 & 7.72 \\
\hline
\end{tabular}

${ }^{\mathrm{a}}$ Reference 36.

${ }^{\mathrm{b}}$ Reference 31 .

${ }^{\mathrm{c}}$ Taken from Refs. $62(a), 79(B)$, and $80\left(E_{b}\right)$.
TABLE V. Binding properties of fcc Al from pseudopotential (PP) and FP-LAPW all-electron calculations. The first column indicates the exchange-correlation functional employed. Shown are the lattice constant $a$, the bulk modulus $B$, and the cohesive energy $E_{b}$. The latter includes the spin-polarization energy $E_{\text {spin }}^{\text {at }}$ for the free $\mathrm{Al}$ atom.

\begin{tabular}{lccccc}
\hline \hline XC & Method & $a(\AA)$ & $B(\mathrm{GPa})$ & $E_{b}(\mathrm{eV})$ & $E_{\text {spin }}^{\text {at }}$ \\
\hline LDA & PP & 3.97 & 80 & 4.09 & 0.15 \\
LDA & LAPW & 3.98 & 84 & 4.07 & \\
LDA & LAPW ${ }^{\mathrm{a}}$ & 3.98 & 83.9 & & \\
PBE & PP & 4.05 & 73 & 3.54 & 0.19 \\
PBE & LAPW & 4.04 & 78 & 3.60 & \\
PBE & LAPW & \\
Experiment & b & 4.04 & 77.3 & & \\
\hline
\end{tabular}

${ }^{\mathrm{a}}$ Reference 74 .

${ }^{\mathrm{b}}$ Taken from Refs. $76(a, B)$ and $77\left(E_{b}\right)$.

orbitals for the $\mathrm{N} 2 s$, Ga $3 p, 3 d$, and In $4 p, 4 d$ states. The potential is expanded up to angular momentum $L_{\max }^{\text {pot }}=6$ within the augmentation spheres, and into plane waves up to a cutoff energy of $484 \mathrm{Ry}$ in the interstitial region. For the Kohn-Sham orbitals we use $L_{\max }^{\text {basis }}=9$ and plane waves up to 70 Ry.

\section{B. Pseudopotential plane-wave calculations}

For our pseudopotential calculations ${ }^{50}$ we employ normconserving scalar-relativistic pseudopotentials of the Troullier-Martins type. ${ }^{51,52}$ We generate separate sets of pseudopotentials for the LDA and each GGA in order to consistently include the differences in the respective corevalence interactions. ${ }^{53}$ Using the potentials in the fully separable form of Kleinman and Bylander, ${ }^{54}$ we verified the absence of unphysical ghost states. ${ }^{55}$ A basis set with plane waves up to a cutoff energy of $80 \mathrm{Ry}$ is used throughout, the scale set by the Ga $3 d$ states. We note that cutoff energies of $40 \mathrm{Ry}(\mathrm{AlN})$ to $60 \mathrm{Ry}(\mathrm{GaN})$ may turn out adequate in more routine applications, which rarely call for as converged absolute energies as we are aiming at here.

To ensure the transferability of our pseudopotentials we have thoroughly checked the scattering, excitation, and hardness properties of the free pseudoatoms along the lines discussed in Ref. 32. For Ga and In our tests indicate similar

TABLE VI. Binding properties of $\alpha$-Ga. As for Table V. For the pseudopotential calculations, $c / a$ and $b / a$ ratios as found in the FP-LAPW calculations were used. Internal parameters are given in Table VII.

\begin{tabular}{|c|c|c|c|c|c|}
\hline $\mathrm{XC}$ & Method & $a(\AA)$ & $B(\mathrm{GPa})$ & $E_{b}(\mathrm{eV})$ & $E_{\text {spin }}^{\text {at }}(\mathrm{eV})$ \\
\hline LDA & PP & 4.44 & 67 & 3.43 & 0.15 \\
\hline LDA & LAPW & 4.44 & 64 & 3.46 & \\
\hline PBE & PP & 4.59 & 49 & 2.69 & 0.18 \\
\hline PBE & LAPW & 4.59 & 53 & 2.71 & \\
\hline \multicolumn{2}{|c|}{ Experiment $^{\mathrm{a}}$} & 4.510 & 61.3 & 2.81 & \\
\hline
\end{tabular}

Taken from Refs. $63(a, B)$ and $77\left(E_{b}\right)$. 
TABLE VII. Equilibrium unit-cell parameters of $\alpha$-Ga calculated with the FP-LAPW method.

\begin{tabular}{lcccc}
\hline \hline & $c / a$ & $b / a$ & $u / a$ & $v / a$ \\
\hline LDA & 1.691 & 0.997 & $0.080(1)$ & 0.157 \\
PBE & 1.690 & 0.993 & $0.080(3)$ & 0.157 \\
Experiment $^{\mathrm{a}}$ & 1.692 & 0.997 & 0.079 & 0.153 \\
\hline \hline
\end{tabular}

${ }^{\mathrm{a}}$ Taken from Ref. 63.

transferability for pseudopotentials that treat the Ga $3 d$ or In $4 d$ states either as core states or as valence states. Nevertheless we find that these two different approaches lead to significantly different predictions in calculations of the bulk properties of $\mathrm{GaN}$ and $\mathrm{InN}$. As a more direct test of the pseudopotentials' transferability we present in Sec. II C a comparison of the bulk properties of the group-III nitrides as calculated within the pseudopotential and the all-electron framework (see Sec. III A for a detailed account of our allelectron data). We so examine the role of the different approximations for the core states that are commonly in use.

In these tests we establish excellent agreement with our all-electron data, i.e., transferability, for the following set of pseudopotentials, which we further use in our comparison of the LDA and the different GGA functionals presented in Sec. III: For Al we include partial core corrections to treat nonlinear core-valence exchange-correlation explicitly. ${ }^{56,57}$ For $\mathrm{Ga}$ and In we include the $3 d$ and $4 d$ states as valence states, and use nonlocal projectors for the $s, p, d$ components together with a norm-conserving $f$ component as the local potential. $^{57}$ For $\mathrm{Al}$ and $\mathrm{N}$ we use nonlocal $s, p$ components with the $d$ component as the local potential. ${ }^{57}$ We note that the more usual approach for $\mathrm{Ga}$ and In has been to include only the $s, p$, and $d$ components, and to choose the $s$ component as the local potential to avoid ghost states. This point is discussed in more detail in the following section.

\section{Accurate pseudopotentials}

In the following we discuss the transferability of pseudopotentials in actual calculations of the cubic AlN, GaN, and InN crystals. We use the difference between our all-electron results and the pseudopotential results as a measure of the transferability, and examine the pseudopotentials within both the LDA and the PBE-GGA. Our aim is to obtain a set of pseudopotentials whose uncertainties are clearly smaller than

TABLE VIII. Binding properties of In. As for Table V. For the pseudopotential calculations, $c / a$ ratios as found in the FP-LAPW calculations were used.

\begin{tabular}{lcccccc}
\hline \hline XC & Method & $a(\AA)$ & $c / a$ & $B(\mathrm{GPa})$ & $E_{b}(\mathrm{eV})$ & $E_{\text {spin }}^{\text {at }}(\mathrm{eV})$ \\
\hline LDA & PP & 4.63 & & 46 & 3.05 & 0.13 \\
LDA & LAPW & 4.58 & 1.025 & 50 & 3.08 & \\
PBE & PP & 4.76 & & 35 & 2.31 & 0.16 \\
PBE & LAPW & 4.77 & 1.018 & 34 & 2.34 & \\
Experiment & ${ }^{a}$ & 4.59 & 1.076 & 41 & 2.52 &
\end{tabular}

Taken from Refs. $78(a, c / a)$ and $77\left(B, E_{b}\right)$.
TABLE IX. Binding properties of the $\mathrm{N}_{2}$ dimer from pseudopotential (PP) and FP-LAPW all-electron calculations. The first column indicates the exchange-correlation functional employed. Shown are the bond length $d$, the frequency of the fundamental mode $\nu$, and the binding energy $E_{b}$. The latter includes a spin correction $E_{\text {spin }}^{\text {at }}$ for the free $\mathrm{N}$ atom.

\begin{tabular}{lccccc}
\hline \hline XC & Method & $d(\AA)$ & $\nu(\mathrm{THz})$ & $E_{b}(\mathrm{eV})$ & $E_{\text {spin }}^{\text {at }}$ \\
\hline LDA & PP & 1.085 & 71.1 & 11.75 & 3.03 \\
LDA & LAPW & 1.095 & 71.9 & 11.57 & \\
LDA & Other $^{\mathrm{a}}$ & 1.096 & 71.4 & 11.58 & \\
PBE & PP & 1.095 & 69.7 & 10.69 & 3.12 \\
PPE & LAPW & 1.102 & 70.6 & 10.49 & \\
PBE & Other $^{\text {a }}$ & 1.103 & & 10.54 & \\
Experiment & & 1.098 & 70.7 & $9.76^{\mathrm{c}}$ & \\
\hline \hline
\end{tabular}

${ }^{a}$ All-electron results from Refs. $15\left(d, E_{b}\right)$ and $18(\nu)$.

${ }^{\mathrm{b}}$ Taken from Ref. 75 .

${ }^{\mathrm{c}}$ Without zero-point vibrational energy, the value is $9.91 \mathrm{eV}$.

the differences between the LDA and the GGA results obtained from all-electron calculations.

Ideally, perfectly transferable pseudopotentials would give the same results as all-electron calculations. In practice, the transferability is limited by the approximations on which the pseudopotential approach relies: (1) The frozen-core approximation which eliminates the (chemically inert) core electrons so that only chemically active valence electrons are treated explicitly. (2) The transformation of the all-electron into the pseudo wave functions which removes the nearnuclear nodes, but should not affect the wave functions in the bonding region. (3) The nonlinear core-valence exchangecorrelation interaction is treated approximately, either linearized and included in the pseudopotential, or explicitly with the help of a partial core density. For the cation pseudopotentials the following, increasingly sophisticated, approaches may be used.

(i) The $s p$ valence approach, where $\mathrm{Al}, \mathrm{Ga}$, and $\mathrm{In}$ are considered as trivalent ions $\left(\mathrm{Al}^{3+}, \mathrm{Ga}^{3+}, \mathrm{In}^{3+}\right)$, and the nonlinear core-valence exchange-correlation interaction is linearized (i.e., approximately included in the pseudopotential operator). This approach is compuationally simplest and

TABLE X. Heat of formation, $\Delta H_{\mathrm{f}}$, and lattice constant $a$ for cubic group-III nitrides calculated with the LDA and different GGA's as described in Sec. III B.

\begin{tabular}{lcccccc}
\hline \hline & \multicolumn{3}{c}{$\Delta H_{\mathrm{f}}(\mathrm{eV} /$ pair $)$} & \multicolumn{3}{c}{$a(\AA)$} \\
& $\mathrm{AlN}$ & $\mathrm{GaN}$ & $\mathrm{InN}$ & $\mathrm{AlN}$ & $\mathrm{GaN}$ & $\mathrm{InN}$ \\
\hline LDA & -3.38 & -1.38 & -0.19 & 4.33 & 4.47 & 4.95 \\
PBE & -2.70 & -0.71 & 0.35 & 4.40 & 4.57 & 5.06 \\
BLYP & -3.17 & -0.56 & 0.47 & 4.42 & 4.61 & 5.12 \\
revPBE & -2.49 & -0.48 & 0.57 & 4.41 & 4.59 & 5.10 \\
RPBE & -2.47 & -0.46 & 0.58 & 4.41 & 4.60 & 5.11 \\
Expt. & $-3.25^{\mathrm{a}}$ & $-1.27^{\mathrm{a}}$ & $-0.32^{\mathrm{a}}$ & & & \\
& $-3.24^{\mathrm{b}}$ & $-1.15^{\mathrm{b}}$ & $-0.18^{\mathrm{b}}$ & & & \\
\hline \hline
\end{tabular}

${ }^{\mathrm{a}}$ Using Eq. (2) with the values for $E_{b}$ from Tables II-IX.

${ }^{\mathrm{b}}$ Reference 81 , at $T=0 \mathrm{~K}(\mathrm{AlN})$ and $298 \mathrm{~K}(\mathrm{GaN}, \mathrm{InN})$. 
well used for III-V semiconductors with $\mathrm{P}$ or As anions instead of N. It treats all states up to the $\mathrm{Al} 2 p$, Ga $3 d$, and In $4 d$ levels as core states.

(ii) The $s p$ valence $+N L C V X C$ approach, where nonlinear core-valence exchange-correlation (NLCV XC) is included explicitly with the help of a partial core density (i.e., the valence and core electron densities add to the total electron density used to determine the exchange-correlation potential and energy).

(iii) The $d+s p$ valence approach for $\mathrm{Ga}$ and $\mathrm{In}$, where the $\mathrm{Ga} 3 d$ and the In $4 d$ states are treated as valence states, considering $\mathrm{Ga}^{13+}$ and $\mathrm{In}^{13+}$ ions. This takes into account the interactions of the closed Ga or In semicore $d$ shells and the nitrogen $2 s$ shell, which is clearly indicated by the calculated Kohn-Sham band structures within the LDA, ${ }^{35}$ but also within the GGA. ${ }^{32}$

In LDA calculations, the $s p$ valence approach is known to yield an accurate description of the structural and elastic properties for $\mathrm{AlN}$, but can lead to lattice parameters of GaN and InN by more than 5\% smaller than in all-electron calculations. This shortcoming is much corrected when the $s p$ valence + NLCV XC approach is employed, as it has been done in LDA studies of dynamical and dielectric properties of GaN (Ref. 3) and AlN/GaN/InN interfaces. ${ }^{58,59}$ The nonlinear core-valence exchange correlation of the LDA has thus a significant effect in $\mathrm{GaN}$ and $\mathrm{InN}$, unlike, for instance, in GaAs. ${ }^{60}$ On the other hand, the reported LDA values for the $\mathrm{GaN}$ lattice constant obtained in this way still vary from less than $4.40 \AA$ to $4.51 \AA$, whereas all-electron calculations give $^{36} 4.46 \AA$ and experimental data range from 4.50 to 4.53 $\AA .{ }^{32}$ Use of the $d+s p$ valence approach has been found essential to obtain lattice parameters in full accordance with all-electron calculations, ${ }^{36}$ and for the study of bulk impurities $^{5}$ and surface ${ }^{12}$ or interface structures. ${ }^{41}$

For the GGA, previous pseudopotential studies give only an incomplete view on the influence of the core electrons on the pseudopotential's transferability. For GaN, Miotto et al. employed the $s p$ valence and $s p$ valence + NLCV XC approach, ${ }^{34}$ obtaining a lattice parameter of $4.45 \AA$ for the PBE-GGA. This value is nearly unchanged compared to the LDA value of $4.46 \AA$, but is substantially smaller than the (only published) all-electron value for the PBE-GGA of 4.57 $\AA$ obtained by van Schilfgaarde et al. ${ }^{31}$ Stampfl and Van de Walle used the $d+s p$ valence approach in their study of the PW91-GGA (which is expected to produce similar results as the PBE-GGA). They found a lattice parameter of $4.59 \AA$, significantly larger than the result of Miotto et al., and in reasonable agreement with the all-electron result for the PBE-GGA of Ref. 31. The treatment of the Ga $3 d$ and In $4 d$ states as valence states thus appears to increase the calculated lattice parameters, consistent with a closed-shell repulsion between the cation semicore $d$ shell and the nitrogen $2 s$ shells. On the other hand, their PW91-GGA cohesive energies for $\mathrm{GaN}$ and $\mathrm{InN}$, but not $\mathrm{AlN}$, are more than $0.7 \mathrm{eV} /$ pair smaller than the experimental values. No such underestimate was found in the PBE-GGA study of Zoroddu et al., ${ }^{33}$ where the semicore $d$ states were also treated as valence states, but ultrasoft pseudopotentials were used instead of norm-conserving pseudopotentials. Neither of the different findings for the cohesive energies has yet been confirmed by an all-electron calculation.

In the following we provide a more complete and systematic assessment of the transferability of (norm-conserving) pseudopotentials for the group-III nitrides within both the LDA and the PBE-GGA. We therefore construct and apply the different cation pseudopotentials according to the abovementioned approaches (i)-(iii). In addition, we examine the influence of the local component of the Ga and In pseudopotentials, which we find to affect the results for $\mathrm{GaN}$ and InN significantly. The local part of the pseudopotential governs the behavior of the partial waves with higher angular momentum, for which the norm-conservation constraint is usually not enforced when constructing the pseudopotential. In the Kleinman-Bylander form of the pseudopotentials used here, the local potential also affects the scattering properties for the low angular-momentum components; in particular, it must be chosen such that no ghost states appear. We shall focus on the $d+s p$ valence approach, where we take the local potential as the $s$-like component of the underlying (semilocal) pseudopotential, in order to avoid ghost states that would occur if the $p$ - or $d$-like components were chosen instead. Alternatively we set the local potential to an $f$-like component, which we constructed in addition to the usual $s, p$, and $d$ components of the pseudopotential. ${ }^{57}$

Table I lists our results for the lattice constant, cohesive energy, and heat of formation of the group-III nitrides calculated with the different pseudopotentials. Comparing with the data from our all-electron FP-LAPW calculations we observe the following.

(1) For AlN, the $s p$ valence + NLCV XC Al pseudopotentials yield results in very good agreement with our allelectron data. The use of pseudopotentials with the $s p$ valence approach with linearized core-valence exchange correlation gives slightly too small (by $\approx 1 \%$ for the LDA) lattice parameters, but does not significantly affect the cohesive energies or heats of formation.

(2) For GaN, the $s p$ valence Ga pseudopotentials overestimate the bond strength compared to the all-electron calculations. This is seen from the underestimate in the LDA lattice parameter of $\approx 4 \%$, and the overestimate in the cohesive energy and the heat of formation of more than $1 \mathrm{eV}$. For the PBE-GGA the errors are smaller, but still noticeable. These errors are much reduced with $s p$ valence + NLCV XC pseudopotentials, to within $\approx 1 \%$ for the lattice parameters and $\approx 0.3 \mathrm{eV}$ for the cohesive energies and heats of formation. Turning to the $d+s p$ valence Ga pseudopotentials, the Ga-N bond strength appears reduced, consistent with a closed-shell repulsion between the now included Ga $3 d$ and $\mathrm{N} 2 s$ valence states. The results are in close agreement with the all-electron data if the $f$-like local potential is used. By contrast, with the $s$-like local potential the lattice parameter tends to be overestimated, and the cohesive energy and the heat of formation underestimated, similar to the results found in Ref. 32.

(3) For InN, the $s p$ valence and the $s p$ valence + NLCV $\mathrm{XC}$ In pseudopotentials both severely underestimate the lattice parameter and overestimate the cohesive energy and heat 
of formation. Including the $4 d$ states yields these quantities in close agreement with the all-electron data when the $f$-like local potential is used. For the $s$-like local potential the In-N bonds are again spuriously weakened, as in the case of GaN and as found in Ref. 32.

Our LDA results for the $d+s p$ valence Ga and In potentials agree reasonably well with those by Satta et al. ${ }^{41}$ who used ultrasoft pseudopotentials (including the Ga $3 d$ and In $4 d$ states), although we note that their lattice constants and cohesive energies for $\mathrm{GaN}$ and $\mathrm{InN}$ differ slightly more from our all-electron FP-LAPW data than for our pseudopotentials with $f$-like local potentials.

We interpret the better transferability achieved for GaN and $\mathrm{InN}$ by using $f$-like local potentials to be due to a better description of the higher angular-momentum components of the electron density by the $f$-like pseudopotential component, which is less repulsive than the $s$ component in the Ga or In core region. High angular-momentum functions with respect to the Ga or In sites are not explicitly considered when constructing the pseudopotentials, but will appear in the GaN and InN crystals, where the density about the Ga and In ions becomes "polarized" due to the formation of the bonds with the neighboring nitrogen atoms (this point has been also raised in Ref. 61).

Regarding the N pseudopotential we have verified that an explicit account of nonlinear core-valence exchange correlation does not affect our results significantly. We noted, however, an overestimate of the binding energy of the $\mathrm{N}_{2}$ dimer by about $0.1 \mathrm{eV} /$ atom compared to all-electron calculations (see Table IX), which is propagated also into the calculated heat of formation. This can be attributed to the overlapping core regions of our $\mathrm{N}$ pseudopotentials, for which we used cutoff radii of $r_{s, p, d}^{\mathrm{ps}, \mathrm{N}}=1.5$ bohr. For the $\mathrm{N}_{2}$ dimer with its short bond length of $\approx 2$ bohr, this leads to overlapping core regions of the pseudopotentials and thus an error in the effective potential and total energy. We have checked that decreasing $r_{s, p, d}^{\mathrm{ps}, \mathrm{N}}$ to $1.0 \mathrm{bohr}$ eliminates the core overlap, yielding a perfect agreement of the pseudopotential and allelectron results for $\mathrm{N}_{2}$. The corresponding $\mathrm{N}$ pseudopotentials would require a plane-wave cutoff much larger than 80 Ry (that is, the scale set by the Ga $3 d$ states), rendering calculations computationally much costlier. We thus consider the small overlap error as acceptable.

Taking a practial viewpoint and comparing our pseudopotentials with the experimental data, Table I shows that a good description of GaN can be attained by using $d+s p$ valence pseudopotentials with an $s$-like local potential (as done in the previous studies) ${ }^{5,12}$ For $\mathrm{InN}$, use of the $f$-like local potential is necessary. Judged from the crystal properties in Table I, an equally good description might be accomplished, in a computationally simpler way, by applying $s p$ valence + NLCV $\mathrm{XC}$ pseudopotentials together with the PBE-GGA. However it remains to be seen whether such an approach works reliably in other, more realistic $\mathrm{GaN}$ or $\mathrm{InN}$ nitride systems.

In summary, the consistently best agreement with the calculated all-electron properties of the group-III nitrides is achieved by using $s p$ valence + NLCV XC pseudopotentials for $\mathrm{Al}$, and $d+s p$ valence pseudopotentials for $\mathrm{Ga}$ and $\mathrm{In}$ with an $f$-like local component. The residual errors for AlN,
$\mathrm{GaN}$, and $\mathrm{InN}$ are then less than $1 \%$ for the lattice parameter, $0.12 \mathrm{eV} /$ pair for the cohesive energy, and $0.20 \mathrm{eV}$ for the heat of formation.

\section{RESULTS FOR THE LDA AND THE GGA}

We have calculated the cohesive properties of cubic AlN, $\mathrm{GaN}, \mathrm{InN}$, and the related elements within the LDA and the PBE-GGA, employing the all-electron FP-LAPW approach initially. Using the highly transferable pseudopotentials discussed in Sec. II C we have obtained equivalent results in pseudopotential plane-wave calculations. Tables II-VIII collect our calculated lattice parameters, bulk moduli, and cohesive energies. Our structural data for the solids agree with those reported by other authors using FP-LMTO (Ref. 31) or FP-LAPW (Ref. 36) all-electron techniques. For the nitrogen dimer (Table IX) we see a gratifying agreement of the results of our supercell calculation and those of cluster calculations. ${ }^{30}$ In the following we first focus in detail on the different performance of the LDA and PBE-GGA. We then extend our discussion to the revised PBE-GGA's and the BLYP-GGA functionals. The results for the latter were obtained in the pseudopotential approach.

\section{A. Cohesive properties for the PBE-GGA}

\section{Structural properties}

For the group-III nitride compounds (Tables II-IV) we find the PBE-GGA to increase the lattice parameters by $1.6 \%$ (AlN) to $2.3 \%$ (InN) compared to the LDA values. Correspondingly, the bulk moduli within the PBE-GGA are lowered by up to $15 \%$ (InN). Compared to experimental values this means that the PBE-GGA somewhat overestimates the lattice parameters, where the overestimate is of similar magnitude as the underestimate found for the LDA. The bulk moduli are underestimated by the PBE-GGA by up to $10 \%$ $(\mathrm{InN})$, whereas the LDA yields values slightly larger than the experimental values. While the available experimental structural or elastic data for the (cubic) nitrides are certainly not as firm as for other semiconductors, ${ }^{62}$ this behavior of the PBE-GGA conforms with the usual tendency of GGA's to expand the crystal equilibrium volume relative to the LDA, often somewhat beyond the experimental values. Correspondingly, the PBE-GGA bulk moduli turn out too small, following a trend also noted in other semiconductors. ${ }^{27,28}$

For the elemental metals (Tables V-VIII), we observe that the PBE-GGA likewise leads to a more expanded lattice than in the LDA. For Al it yields the lattice parameter and bulk modulus in good accordance with experiment, improving over the LDA. For the orthorhombic $\alpha$-Ga, the PBEGGA overestimates the equilibrium volume by $\approx 5 \%$, for In by $\approx 6 \%$. This is of similar magnitude as the underestimates we find within the LDA, $\approx 4 \%$ for $\alpha$-Ga and $\approx 5 \%$ for In. The PBE-GGA and LDA both give the unit-cell parameters $c / a$ and $b / a$ of $\alpha-\mathrm{Ga}$, and the positions of the four basis atoms $( \pm u, 0, \pm v)$ and $\left(\frac{1}{2} \pm u, 0, \frac{1}{2} \mp v\right)$, in close agreement with the respective low-temperature experimental data given in Ref. 63. For tetragonal In, the calculated $c / a$ ratios turn out somewhat too low for both the PBE-GGA and the LDA. 
The bulk moduli are again underestimated by the PBE-GGA and overestimated by the LDA. This outcome conforms with the respective overestimates and underestimates of the equilibrium volumes. While not taken into account here, we estimate that zero-point vibrations would raise the calculated equilibrium volumes by less than $2 \%$ for the $\mathrm{Al}, \mathrm{Ga}$, and $\mathrm{In}$ metals. ${ }^{64}$ We thus conclude that the PBE-GGA performs slightly better than the LDA for $\mathrm{Al}$, whereas the LDA gives a better description of the equlibrium volumes of $\mathrm{Ga}$ and $\mathrm{In}$.

\section{Cohesive energies}

Concerning cohesive energies of solids and binding energies of molecules with respect to free atoms, the LDA is known to yield systematically too large values, producing an "overbinding." This is seen for the group-III nitrides as well, where we find an overestimate (per atom) on the order of $0.7-0.9 \mathrm{eV}$, as compared to $0.5-0.7 \mathrm{eV}$ for the metals, and 1 $\mathrm{eV}$ for the $\mathrm{N}_{2}$ molecule (Table IX). The PBE-GGA yields binding energies to within $\approx 0.2 \mathrm{eV} /$ atom for the bulk groupIII nitrides and the elemental metals. We note a tendency towards underbinding for the heavier group-III species. However the $\mathrm{N}_{2}$ molecule is still overbound by the PBEGGA, by about $0.4 \mathrm{eV} /$ atom. Altogether the cohesive energies turn out fairly accurate in the PBE-GGA which thus corrects the LDA's overbinding as in other semiconductors and metals.

\section{B. Heat of formation and comparison of different GGA's}

As a further test of the PBE-GGA we now consider the heat of formation of the group-III nitrides. We note that the value of the heat of formation constrains the allowed range of the chemical potential for adding or removing a constituent cation or $\mathrm{N}$ atoms to a group-III nitride system and is thus of importance in determining the thermodynamic stability of, e.g., bulk impurities or surface structures. In the values reported here we exclude contributions from the the vibrational zero-point energies of the different compounds, which we estimate to cancel out to within $0.1 \mathrm{eV} /$ pair. $^{65}$

From our results given in Table $\mathrm{X}$ we see that the PBEGGA underestimates the heat of formation of the group-III nitrides when compared to experiment. In particular, InN is wrongly predicted to be endothermic, i.e., thermodynamically unstable. By contrast, the LDA values agree consistently better with the experimental data. ${ }^{66}$ This seems surprising in view of the favorable performance of the PBEGGA for the cohesive or binding energies of the individual compounds: Whereas in LDA the pronounced overbinding in the $\mathrm{N}_{2}$ molecule and the nitride and elemental solids largely cancels out, the respective, much smaller errors of the PBEGGA add up.

A different behavior has been observed for other III-V semiconductors like AlAs and GaAs, where the PBE or the closely related PW91-GGA's describe the heat of formation fairly accurately, as does the LDA. ${ }^{67}$ This outcome is plausible for these GGA's since their cohesive energies are accurate for the compound and elemental solids. For the case of the LDA it is usually understood as a cancellation of the errors in the free-atom energies which dominate those in the

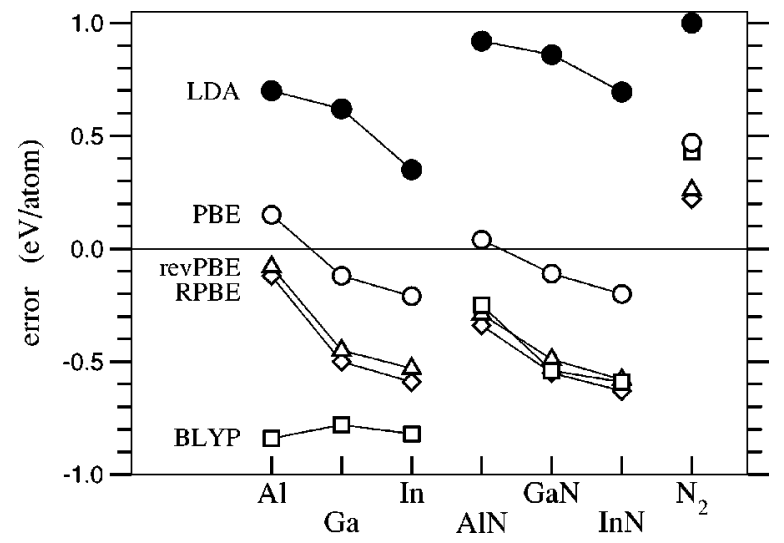

FIG. 1. Calculated cohesive energies for cubic AlN, GaN, InN and the related elemental metals, and binding energy of the $\mathrm{N}_{2}$ dimer. Shown are the deviations with respect to the experimental values (see Tables II-IX) for the LDA (filled circles) and the PBE(open circles), revPBE- (triangles), RPBE- (diamonds), and BLYP(squares) GGA exchange-correlation functionals discussed in Sec. III B. Lines are only meant to group the data points for the elemental metals and the nitride compounds.

cohesive energies. The problem of the PBE-GGA in the case of the group-III nitrides might therefore well arise from the fact that it still overestimates the binding energy of $\mathrm{mol}$ ecules like $\mathrm{N}_{2}$.

In the following we therefore examine whether gradient corrected functionals other than the PBE-GGA lead to a better account of the binding energy of molecules and, in turn, also of the heat of formation for the group-III nitrides. We explore several different GGA functionals.

(i) The BLYP-GGA which has been well used for molecular systems, but has received less attention for solid-state applications. It consists of Becke's GGA for exchange ${ }^{39}$ and that of Lee et al. ${ }^{40}$ for correlation.

(ii) The revPBE-GGA of Zhang and Yang, ${ }^{37}$ who use the same analytic form for the exchange part as the PBE functional, but determine its so-called $\kappa$ parameter empirically from atomic exchange energies rather than by enforcing the Lieb-Oxford bound on the exchange energy locally, one of the nonempirical constraints used in original derivation of the PBE functional. Thereby they arrive at improved molecular binding energies.

(iii) The RPBE-GGA of Hammer et al. ${ }^{38}$ who express the exchange part differently to the PBE, but such that the same formal constraints including the local Lieb-Oxford bound are fulfilled while a performance similar to the revPBE is attained. Using the RPBE-GGA, adsorption energies for several molecules on transition-metal surfaces could be improved compared to the PBE-GGA. ${ }^{38}$ The correlation component in both the revPBE and the RPBE-GGA is identical to that of the original PBE-GGA.

Using these different GGA's we have recalculated the binding energies of the $\mathrm{N}_{2}$ dimer and the solids. The results are shown in Fig. 1. Indeed, the overbinding of the nitrogen molecule found for the PBE is much corrected for by the revPBE and the RPBE-GGA's. For the BLYP-GGA the correction is marginal. On the other hand, Fig. 1 shows that the alternative GGA functionals all lead to marked underbinding 
in the bulk crystals. This trend is also reflected by the lattice constants shown in Table $\mathrm{X}$, which turn out clearly too large compared to experimental values, an effect that has been recently demonstrated for other solids as well. ${ }^{68}$ We note that the revPBE and the RPBE-GGA's lead to very similar results, underestimating the cohesive energies by up to $0.6 \mathrm{eV} /$ atom in the case of In and InN. While the BLYP-GGA performs similarly to the revPBE and RPBE-GGA for the bulk nitrides, it produces an even more pronounced underbinding for the elemental metals, by up to $0.8 \mathrm{eV} /$ atom. Figure 1 clearly shows that only the PBE-GGA yields the cohesive energies consistently close to the experimental data, i.e., to within $0.2 \mathrm{eV} /$ atom. In Table $\mathrm{X}$ we show the corresponding heats of formation. As a consequence of the underestimate of the cohesive energies, the revPBE and RPBE-GGA's lead to an even larger underestimate of the formation enthalpies of the group-III nitrides than the PBE-GGA. For the BLYPGGA this is also the case for $\mathrm{GaN}$ and $\mathrm{InN}$; in the case of AlN, it yields an accurate value due to a cancellation of the large errors in the cohesive energies of the $\mathrm{Al}$ and $\mathrm{AlN}$ bulk crystals.

In effect, the revPBE-, RPBE-, and BLYP-GGA's yield a less realistic description of the group-III metal and group-III nitride bulk crystals than the PBE-GGA, even though they succeed to improve for the $\mathrm{N}_{2}$ dimer. We are led to the conclusion that none of the GGA's investigated here describes the heat of formation of the group-III nitrides satisfactorily, whereas the LDA yields a reasonably accurate account.

\section{Qualitative analysis of the different GGA's}

In the following we discuss how the different binding energies for the different GGA's may be related to the dependence of these functionals on the density. Our aim is to better understand the different behavior of the PBE-GGA and the revPBE-, RPBE-, and BLYP-GGA's in the $\mathrm{N}_{2}$ molecule and the solids that we have observed in the preceding section. To this end we analyze the difference of the GGA and LDA ground-state total energies, which is given, correct to second order in the difference of the self-consistent LDA and GGA densities,${ }^{53}$ by

$$
E_{\mathrm{tot}}^{\mathrm{GGA}}\left[n^{\mathrm{GGA}}\right]-E_{\mathrm{tot}}^{\mathrm{LDA}}\left[n^{\mathrm{LDA}}\right] \simeq \Delta E_{\mathrm{xc}}^{\mathrm{GGA}}\left[n^{\mathrm{LDA}}\right]
$$

where the right-hand side represents the difference between the exchange-correlation energies $E_{\mathrm{xc}}^{\mathrm{LDA}, \mathrm{GGA}}$ taken at the self-consistent LDA density $n^{\mathrm{LDA}}$,

$$
\Delta E_{\mathrm{xc}}^{\mathrm{GGA}}[n]=: E_{\mathrm{xc}}^{\mathrm{GGA}}[n]-E_{\mathrm{xc}}^{\mathrm{LDA}}[n], \quad n=n^{\mathrm{LDA}} .
$$

Such "post-LDA" calculations, supported by the variational principle (if the external potential is held fixed), accurately reflect the full differences between self-consistent LDA and GGA total energies. Therefore we can examine the GGA corrections to LDA cohesive energies using

$$
E_{b}^{\mathrm{GGA}}-E_{b}^{\mathrm{LDA}} \simeq \Delta E_{\mathrm{xc}}^{\mathrm{GGA}}\left[n^{\text {atoms }}\right]-\Delta E_{\mathrm{xc}}^{\mathrm{GGA}}\left[n^{\text {solids }}\right]
$$

TABLE XI. GGA induced changes in the total energy and the binding energy relative to the LDA values, calculated post LDA, i.e., from the change in the exchange-correlation energies using Eqs. (4) and (5). Presented is the case of AlN and the related Al and $\mathrm{N}$ species. The bracketed values show the (mostly dominant) contributions due to gradient corrections for exchange alone. This post-LDA analysis uses the (pseudo-) valence electron densities determined from LDA pseudopotential calculations. Termed full GGA

\begin{tabular}{|c|c|c|c|c|}
\hline & \multicolumn{2}{|c|}{$\begin{array}{c}\Delta E_{\mathrm{xc}}^{\mathrm{GGA}}[\text { Eq. (4)] } \\
(\mathrm{eV} / \text { electron })\end{array}$} & \multicolumn{2}{|c|}{$\begin{array}{l}E_{b}^{\mathrm{GGA}}-E_{b}^{\mathrm{LDA}} \\
(\mathrm{eV} / \text { electron) }\end{array}$} \\
\hline & & & \multirow[t]{2}{*}{$\begin{array}{l}\text { Post LDA } \\
{[\text { Eq. }(5)]}\end{array}$} & \multirow[t]{2}{*}{ Full GGA } \\
\hline & $\mathrm{N}$ atom & $\mathrm{N}_{2}$ molecule & & \\
\hline PBE & $-0.32(-0.92)$ & $-0.27(-0.81)$ & $-0.05(-0.11)$ & -0.106 \\
\hline revPBE & $-0.42(-1.02)$ & $-0.34(-0.88)$ & $-0.08(-0.14)$ & -0.147 \\
\hline RPBE & $-0.43(-1.04)$ & $-0.36(-0.90)$ & $-0.07(-0.14)$ & -0.155 \\
\hline \multirow[t]{2}{*}{ BLYP } & $-0.28(-1.01)$ & $-0.18(-0.89)$ & $-0.10(-0.12)$ & -0.114 \\
\hline & $\mathrm{Al}$ atom & Al crystal & & \\
\hline PBE & $-0.12(-0.53)$ & $-0.01(-0.06)$ & $-0.11(-0.47)$ & -0.18 \\
\hline revPBE & $-0.19(-0.59)$ & $-0.01(-0.06)$ & $-0.18(-0.53)$ & -0.26 \\
\hline RPBE & $-0.20(-0.61)$ & $-0.01(-0.07)$ & $-0.19(-0.54)$ & -0.27 \\
\hline \multirow[t]{2}{*}{ BLYP } & $-0.02(-0.58)$ & $0.42(-0.07)$ & $-0.44(-0.51)$ & -0.51 \\
\hline & $\mathrm{Al}, \mathrm{N}$ atoms & AlN crystal & & \\
\hline PBE & $-0.25(-0.78)$ & $-0.11(-0.51)$ & $-0.14(-0.26)$ & -0.22 \\
\hline revPBE & $-0.33(-0.86)$ & $-0.13(-0.53)$ & $-0.21(-0.33)$ & -0.30 \\
\hline RPBE & $-0.35(-0.88)$ & $-0.13(-0.54)$ & $-0.21(-0.34)$ & -0.31 \\
\hline BLYP & $-0.18(-0.84)$ & $0.05(-0.56)$ & $-0.23(-0.28)$ & -0.29 \\
\hline
\end{tabular}
are the changes found from self-consistent GGA calculations with the respective GGA pseudopotentials. All calculations of $\mathrm{N}_{2}$, and the $\mathrm{Al}$ and $\mathrm{AlN}$ crystals were performed at their experimental structures. 

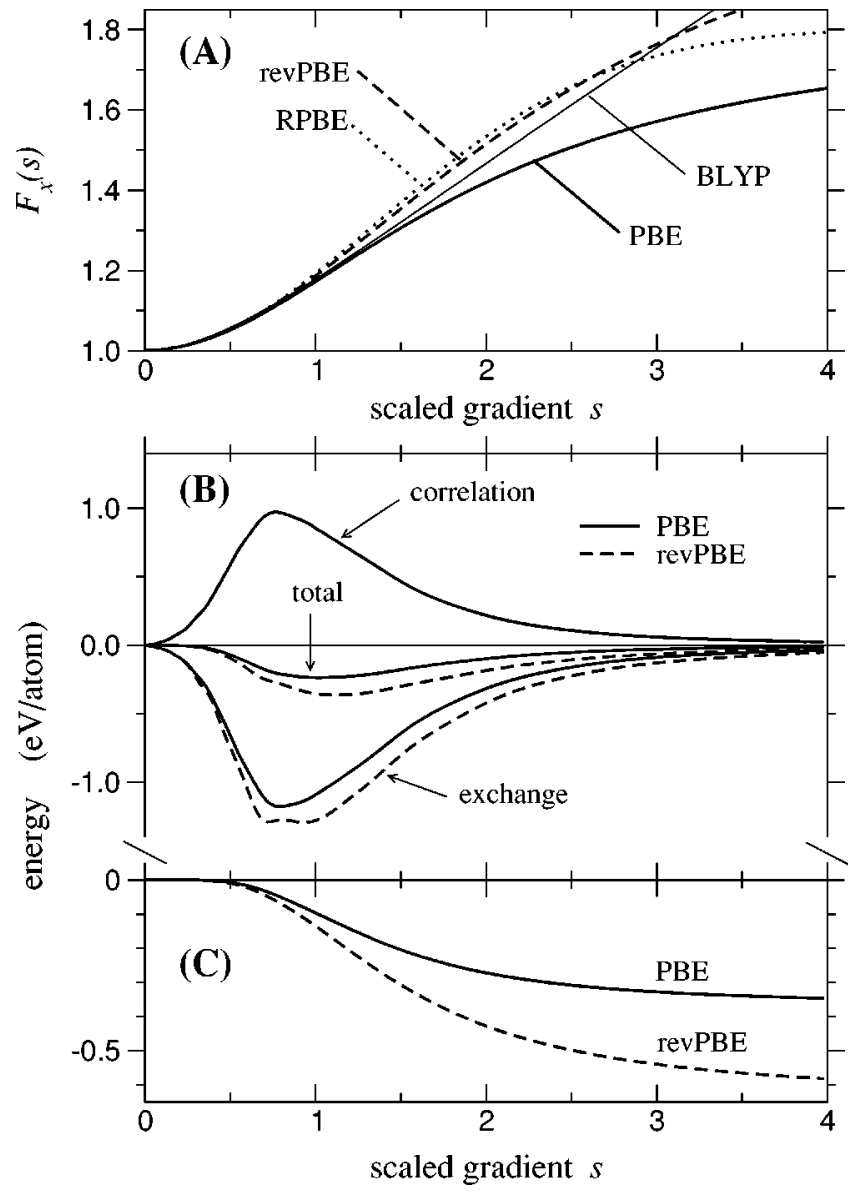

FIG. 2. Graphical view of the GGA induced change of the binding energy relative to the LDA as discussed in Sec. III C. Panel (a) shows the enhancement factor $F_{\mathrm{x}}(s)$ [see Eq. (6)] for the exchange part of the different GGA's as a function of the scaled gradient $s$. Note that $F_{\mathrm{x}}(s)=1$ corresponds to the LDA, and that $F_{\mathrm{x}}^{\mathrm{PBE}, \mathrm{RPBE}}(s) \leqslant 1.803$ by the Lieb-Oxford bound imposed in these two functionals. Panel (b) shows the $s$-decomposed correction to the binding energy due to GGA [see Eq. (7)] for fcc aluminum in the case of the PBE and revPBE. The stronger nonlocality or enhancement in the exchange part of the revPBE leads to larger negative corrections than the PBE. Therefore also the $s$-integrated total change in the binding energy [see Eq. (8)], shown in Panel (c), turns out larger and leads to a lower binding energy for the revPBE-GGA than for the PBE-GGA. Note that contributions from $s_{\sim 2}^{>}$are entirely due to the free aluminum atom and become energetically unimportant for $s_{\sim 4}^{>}$.

and likewise for molecules, where it is useful to consider the corrections per electron in order to compare free atoms and polyatomic compounds.

We have evaluated the GGA corrections Eq. (5) for the valence electrons of the $\mathrm{Al}$ and $\mathrm{N}$ atoms, the $\mathrm{Al}$ and $\mathrm{AlN}$ crystals, and the $\mathrm{N}_{2}$ dimer, using the valence densities from LDA pseudopotential calculations. As can be seen from Table XI the magnitude of the correction $\Delta E_{\mathrm{xc}}^{\mathrm{GGA}}$ (per electron) turns out largest for the free $\mathrm{N}$ and $\mathrm{Al}$ atoms. It decreases when going to the $\mathrm{N}_{2}$ molecule or the AlN crystal, and is nearly zero for the Al bulk metal. In this way the GGA's act to reduce the binding energies compared to the
LDA, and tend to increase the crystal equilibrium volumes. ${ }^{19,70}$ An analogous analysis holds for the Ga and In metals, and the GaN and InN crystals.

To further resolve the differences among the GGA's and relative to the LDA, it is useful to write the exchangecorrelation energy as

$$
E_{\mathrm{xc}}^{\mathrm{LDA}, \mathrm{GGA}}[n]=\left.\int \epsilon_{\mathrm{x}}^{\mathrm{hom}}(n) F_{\mathrm{xc}}^{\mathrm{LDA}, \mathrm{GGA}}(n, s)\right|_{s(\mathbf{r})} ^{n(\mathbf{r})} d^{3} r,
$$

and characterize the different GGA's through their enhancement factors $F_{\mathrm{xc}}(n, s)=F_{\mathrm{x}}(s)+F_{\mathrm{c}}(n, s)$, defined with respect to the LDA exchange energy per electron $\epsilon_{x}^{\text {hom }}(n)<0 .{ }^{15}$ Here $s$ is the scaled density gradient of the electron density,

$$
s(\mathbf{r})=\frac{|\boldsymbol{\nabla} n(\mathbf{r})|}{2\left(3 \pi^{2}\right)^{1 / 3} n^{4 / 3}(\mathbf{r})} .
$$

In the limit of a homogeneous density one has $|\boldsymbol{\nabla} n(\mathbf{r})| \equiv 0$, so that $F_{\mathrm{xc}}^{\mathrm{GGA}}(n, s \rightarrow 0)=F_{\mathrm{xc}}^{\mathrm{LDA}}(n)$ represents the LDA. The different degree to which different GGA's lower (or raise) the exchange-correlation energy can then be understood in terms of $(n, s)$ dependence of the respective $F_{\mathrm{xc}}$. Figure 2 shows the enhancement factor for the exchange part, $F_{\mathrm{x}}(s)$, for the GGA's used in this study. Note that $F_{\mathrm{x}}$ does not depend explicitly on $n$. Corresponding plots, which include the correlation contributions, can be found in Refs. 16 and 30. From Fig. 2 we see that the exchange energy becomes more negative with increasing $s$, where the enhancement factors rise monotonically from $F_{\mathrm{x}}^{\mathrm{GGA}}(s=0)=F_{\mathrm{LDA}}=1$ to the local Lieb-Oxford bound $F_{\mathrm{x}}^{\mathrm{GGA}}(s \gg 1) \rightarrow 1.803$ for the $\mathrm{PBE}$ and $\mathrm{RPBE}$, or beyond it in the revPBE and BLYP functionals. The performance of the different GGA's can now be interpreted as follows: (i) Since the enhancement grows more rapidly for the RPBE-, revPBE-, and BLYP-GGA's these necessarily lead to more negative exchange energies than the PBE-GGA, as Table XI indeed shows. (ii) Since on the average $s$ is larger for atoms than solids, ${ }^{69}$ the exchange part of the GGA's gives a repulsive correction to the LDA binding energy, consistent with our findings in Table XI. (iii) By their larger enhancement factors, the RPBE, revPBE, and BLYPGGA's therefore lead to lower binding energies than the PBE-GGA's. To make this reasoning more explicit, we now inspect the $s$-decomposed correction to the LDA exchange correlation, in terms of the (pseudo-) valence density,

$$
\begin{aligned}
& \Delta E_{\mathrm{xc}}^{\mathrm{GGA}}([n], s) \\
& \quad=\left.\int \epsilon_{\mathrm{x}}^{\mathrm{hom}}(n)\left[F_{\mathrm{xc}}^{\mathrm{GGA}}\left(n, s^{\prime}\right)-F_{\mathrm{xc}}^{\mathrm{LDA}}(n)\right]\right|_{s^{\prime}(\mathbf{r})=s} ^{n(\mathbf{r})} d^{3} r,
\end{aligned}
$$

as proposed in Refs. 21, 38, and 70. The difference between $\Delta E_{\mathrm{xc}}^{\mathrm{GGA}}(s)$ in the solid and the free atoms represents the GGA correction to the binding energy at each $s$ value occurring in both systems. Integration of this difference leads back to Eq. (5), 


$$
\begin{aligned}
E_{b}^{\mathrm{GGA}}-E_{b}^{\mathrm{LDA}} \simeq & \int_{0}^{\infty} \Delta E_{\mathrm{xc}}^{\mathrm{GGA}}\left(\left[n^{\text {atoms }}\right], s\right) \\
& -\Delta E_{\mathrm{xc}}^{\mathrm{GGA}}\left(\left[n^{\text {solids }}\right], s\right) d s .
\end{aligned}
$$

In Fig. 2 we show this decomposition for aluminum. Seen as a function of $s$, the GGA correction to the LDA cohesive energy turns out stronger for the revPBE than for the PBE. This clearly reflects the fact that $F_{\mathrm{x}}^{\mathrm{revPBE}}(s)>F_{\mathrm{x}}^{\mathrm{PBE}}(s)$. We observe that contributions for $s \gtrsim 2$ are entirely due to electron density in the free atom, whereas the Al bulk crystal contributes only for $s \lesssim 2$. Figure 2 further shows that the PBE gradient corrections for correlation partly cancel the gradient corrections for exchange.

We have focused here on the $s$ dependence of the GGA's, which is sufficient for the PBE and the revised PBE-GGA's as their density dependence is the same appearing in $\epsilon_{\mathrm{x}}^{\text {hom }}(n)$ and $F_{\mathrm{c}}(n, s)$. In the BLYP approximation, the correlation energy requires a consideration of the different density dependence as well, which we do not carry through here; since the exchange part of the BLYP approximation is similar to that of the revised PBE-GGA's, it is clear, however, that the additional underestimate of the cohesive energies in the $\mathrm{Al}, \mathrm{Ga}$, and In metals is due to the LYP correlation functional. Finally, we briefly consider the contributions to the GGA induced change in the cohesive energy, which are due to corevalence exchange correlation and spin polarization. From Table XI these can be estimated as the difference between the post-LDA results and the self-consistent full GGA results obtained with the proper GGA pseudopotentials. These differences indicate a further enlargement of the GGA induced lowering of the cohesive and binding energies, and more so for revPBE- and RPBE-GGA's, consistent with our above findings for the valence electrons.

Our analysis demonstrates that, relative to the LDA, the revPBE-, RPBE-, and BLYP-GGA's lead to larger corrections in binding energies than the PBE-GGA. At least for the revPBE and RPBE this is achieved mainly through a more rapid increase of the gradient corrections for exchange when the density gradient becomes larger. In this sense they represent more nonlocal GGA's than the PBE-GGA. Despite the improvements found for molecules, our results for the bulk group-III metals and group-III nitrides indicate that more nonlocal GGA functionals may entail a less accurate description of solids.

\section{SUMMARY AND CONCLUSIONS}

We have investigated the performance of various generalized gradient approximations (GGA's) in density-functional calculations of the cohesive properties of cubic AlN, GaN, and $\mathrm{InN}$, the associated elemental $\mathrm{Al}, \mathrm{Ga}$, and In metals and the $\mathrm{N}_{2}$ molecule. In the first part of this study we have used all-electron FP-LAPW calculations to scrutinize the results from the pseudopotential approach, where previous studies show a significant spread dependent on the construction of the pseudopotentials. Our comparison shows that, in pseudopotential calculations, the semicore $d$ states of Ga and In are best treated as valence electrons in order to achieve the transferability needed for a proper assessment of the LDA and GGA with respect to experimental data. Including an $f$-like local potential for $\mathrm{Ga}$ and $\mathrm{In}$, we arrive at a set of highly transferable pseudopotentials which yield results in excellent agreement with our all-electron data. By contrast, when the $\mathrm{Ga}$ and In semicore $d$ states are treated as core states-but taking into account nonlinear core-valence exchange correlation through partial core densities - the Ga-N and In-N bonds appear stronger and shorter than our all-electron results suggest.

In the second part of our study we examined the effect of the PBE-GGA on the calculated cohesive properties. We find that the lattice parameters are slightly overestimated within the PBE-GGA, and improved with respect to the LDA only in case of $\mathrm{Al}$ and $\mathrm{AlN}$. The PBE-GGA yields cohesive energies within $0.2 \mathrm{eV}$ of the experimental data for the crystalline group-III nitrides and $\mathrm{Al}, \mathrm{Ga}$, and In elemental metals, clearly correcting the overbinding of the LDA for these bulk solids. For the $\mathrm{N}_{2}$ molecule, the binding energy improves as well over the LDA, although it remains overestimated by about $0.6 \mathrm{eV}$ compared to the experimental value. The heats of formation of $\mathrm{AlN}$, GaN, and $\mathrm{InN}$ are given reasonably accurately in the LDA, albeit due to an error cancellation between the overestimates of the bond strengths in both the solids and the $\mathrm{N}_{2}$ molecule. Within the PBE-GGA the heats of formation are underestimated, in particular, $\mathrm{InN}$ is predicted to be endothermic. We attribute this shortcoming mainly to the $\mathrm{N}_{2}$ dimer, where the binding energy is still overestimated, rather than the solids, where the PBE-GGA yields accurate cohesive energies and thus no error cancellation takes place. Using the recent revised PBE-GGA functionals by Zhang and Yang and by Hammer et al. one obtains a more accurate binding energy of the $\mathrm{N}_{2}$ molecule. However we also find that the revised PBE-GGA's as well as the earlier BLYP-GGA significantly underestimate the cohesive energies of the group-III nitride and group-III elemental bulk crystals, where they are clearly less accurate than the PBEGGA. In effect, the revised PBE- and BLYP-GGA's worsen the calculated heats of formation of the group-III nitrides compared to the PBE-GGA. An analysis of the different GGA functionals shows that the reduction of the cohesive energies is due to stronger gradient corrections or nonlocality, in particular, in the exchange energy component of these functionals. While the increased nonlocality improves the description of molecular binding energies, our results show that it worsens the description of the cohesive energies of solids. We regard the group-III nitride/ $\mathrm{N}_{2}$ systems as a representative test case, which illustrates the difficulties present GGAtype (explicit) density functionals still have with being consistently accurate for both molecules and solids. For molecules it has been shown that further improvements are possible with orbital dependent (implicit) density functionals, such as hybrid functionals that include exact exchange $^{71,72}$ or meta-GGA's that depend on the orbital kinetic-energy density. ${ }^{73,74}$ It remains to be seen whether these improvements carry over to solid systems. 
*Present address: Department of Physics and Astronomy, Northwestern University, Evanston, Illinois.

${ }^{1}$ F.A. Ponce and D.P. Bour, Nature (London) 386, 351 (1997).

${ }^{2}$ K. Kim, W.R.L. Lambrecht, and B. Segall, Phys. Rev. B 53, 16310 (1996).

${ }^{3}$ K. Karch, J.-M. Wagner, and F. Bechstedt, Phys. Rev. B 57, 7043 (1998).

${ }^{4}$ N.E. Christensen and I. Gorczyca, Phys. Rev. B 50, 4397 (1994).

${ }^{5}$ J. Neugebauer and C.G. Van de Walle, in Diamond, SiC and Nitride Wide Bandgap Semiconductors, edited by C.H. Carter, Jr., G. Gildenblat, S. Nakamura, and R.J. Nemanich, Mater. Res. Soc. Symp. Proc. No. 339 (Materials Research Society, Pittsburgh, 1994), p. 687.

${ }^{6}$ J.E. Northrup, L.T. Romano, and J. Neugebauer, Appl. Phys. Lett. 74, 2319 (1999).

${ }^{7}$ P. Boguslawski and J. Bernholc, Phys. Rev. B 56, 9496 (1997).

${ }^{8}$ C.G. Van de Walle, C. Stampfl, and J. Neugebauer, J. Cryst. Growth 189/190, 505 (1998).

${ }^{9}$ I. Gorczyca, A. Svane, and N.E. Christensen, Phys. Rev. B 60, 8147 (1999).

${ }^{10}$ F. Bernardini and V. Fiorentini, Phys. Rev. B 57, 9427 (1998).

${ }^{11}$ J. Neugebauer, T. Zywietz, M. Scheffler, J.E. Northrup, and C.G. Van de Walle, Phys. Rev. Lett. 80, 3097 (1998).

${ }^{12}$ T. Zywietz, J. Neugebauer, and M. Scheffler, Appl. Phys. Lett. 73, 487 (1998).

${ }^{13}$ W. Kohn, A.D. Becke, and R.G. Parr, J. Phys. Chem. 100, 12974 (1996), and references therein.

${ }^{14}$ R.O. Jones and O. Gunnarsson, Rev. Mod. Phys. 61, 689 (1989).

${ }^{15}$ J.P. Perdew, K. Burke, and Y. Wang, Phys. Rev. B 54, 16533 (1996), and references therein.

${ }^{16}$ J.P. Perdew and K. Burke, Int. J. Quantum Chem. 57, 309 (1996).

${ }^{17}$ A.D. Becke, J. Chem. Phys. 96, 2155 (1992).

${ }^{18}$ B.G. Johnson, P.M.W. Gill, and J.A. Pople, J. Chem. Phys. 98, 5612 (1993).

${ }^{19}$ X.J. Kong, C.T. Chan, K.M. Ho, and Y.Y. Ye, Phys. Rev. B 42, 9357 (1990).

${ }^{20}$ M. Körling and J. Häglund, Phys. Rev. B 45, 13293 (1992).

${ }^{21}$ P.H.T. Philipsen and E.J. Baerends, Phys. Rev. B 54, 5326 (1996).

${ }^{22}$ I.-H. Lee and R.M. Martin, Phys. Rev. B 56, 7197 (1997).

${ }^{23}$ J. Baker, M. Muir, and J. Andzelm, J. Chem. Phys. 102, 2063 (1995).

${ }^{24}$ L. Fan and T. Ziegler, J. Am. Chem. Soc. 114, 10 (1992).

${ }^{25}$ B. Hammer, K. W. Jacobsen, and J.K. Nørskov, Phys. Rev. Lett. 70, 3971 (1993).

${ }^{26}$ E. Pehlke and M. Scheffler, Phys. Rev. Lett. 74, 952 (1995).

${ }^{27}$ C. Filippi, D.J. Singh, and C.J. Umrigar, Phys. Rev. B 50, 14947 (1994).

${ }^{28}$ G. Ortiz, Phys. Rev. B 45, 11328 (1992).

${ }^{29}$ D.M. Ceperley and B.J. Alder, Phys. Rev. Lett. 45, 566 (1980); J.P. Perdew and Y. Wang, Phys. Rev. B 45, 13244 (1992).

${ }^{30}$ J.P. Perdew, K. Burke, and M. Ernzerhof, Phys. Rev. Lett. 77, 3865 (1996); 80, 891 (1998).

${ }^{31}$ M. van Schilfgaarde, A. Sher, and A.-B. Chen, J. Cryst. Growth 178, 8 (1997).

${ }^{32}$ C. Stampfl and C.G. Van de Walle, Phys. Rev. B 59, 5521 (1999).

${ }^{33}$ A. Zoroddu, F. Bernardini, P. Ruggerone, and V. Fiorentini, Phys. Rev. B 64, 045208 (2001).

${ }^{34}$ R. Miotto, G.P. Srivastava, and A.C. Ferraz, Phys. Rev. B 59, 3008 (1999); Surf. Sci. 435, 377 (1999).
${ }^{35}$ V. Fiorentini, M. Methfessel, and M. Scheffler, Phys. Rev. B 47, 13353 (1993).

${ }^{36}$ A.F. Wright and J.S. Nelson, Phys. Rev. B 51, 7866 (1995); 50, 2159 (1994).

${ }^{37}$ Y. Zhang and W. Yang, Phys. Rev. Lett. 80, 890 (1998).

${ }^{38}$ B. Hammer, L.B. Hansen, and J.K. Nørskov, Phys. Rev. B 59, 7413 (1999).

${ }^{39}$ A.D. Becke, Phys. Rev. A 38, 3098 (1988).

${ }^{40}$ C. Lee, W. Yang, and R.G. Parr, Phys. Rev. B 37, 785 (1988).

${ }^{41}$ A. Satta, V. Fiorentini, A. Bosin, and F. Meloni, in Gallium Nitride and Related Materials, edited by R.D. Dupuis, I.A. Edmond, F.A. Ponce, and S. Nakamura, Mater. Res. Soc. Symp. Proc. No. 395 (Materials Research Society, Pittsburgh, 1996), p. 515 .

${ }^{42}$ C. Stampfl, C.G. Van de Walle, D. Vogel, P. Krüger, J. Pollmann, Phys. Rev. B 61, 7846 (2000).

${ }^{43}$ F.D. Murnaghan, Proc. Natl. Acad. Sci. U.S.A. 30, 244 (1944).

${ }^{44}$ H.J. Monkhorst and J.D. Pack, Phys. Rev. B 13, 5188 (1976).

${ }^{45}$ M.J. Gillan, J. Phys.: Condens. Matter 1, 689 (1989); J. Neugebauer and M. Scheffler, Phys. Rev. B 46, 16067 (1992).

${ }^{46}$ E.J. Baerends, V. Branchadell, and M. Sodupe, Chem. Phys. Lett. 265, 481 (1997).

${ }^{47}$ D.J. Singh, Plane Waves, Pseudopotentials and the LAPW Method (Kluwer, Dordrecht, 1994).

${ }^{48}$ P. Blaha, K. Schwarz, and J. Luitz, computer code WIEN97 (Vienna University of Technology, Vienna, 1997) [Improved and updated UNIX version of the original copyrighted WIEN code, which was published by P. Blaha, K. Schwarz, P. Sorantin, and S.B. Trickey, Comput. Phys. Commun. 59, 399 (1990)]; B. Kohler, S. Wilke, M. Scheffler, R. Kouba, and C. AmbroschDraxl, ibid. 94, 31 (1996); M. Petersen, F. Wagner, L. Hufnagel, M. Scheffler, P. Blaha, and K. Schwarz, ibid. 126, 294 (2000).

${ }^{49}$ S.H. Wei and A. Zunger, Phys. Rev. B 37, 8958 (1988).

${ }^{50}$ M. Bockstedte, A. Kley, J. Neugebauer, and M. Scheffler, Comput. Phys. Commun. 107, 187 (1997).

${ }^{51}$ M. Fuchs and M. Scheffler, Comput. Phys. Commun. 109, 67 (1999); http://www.FHI-Berlin;MPG.DE/th/fhimd

${ }^{52}$ N. Troullier and J.L. Martins, Phys. Rev. B 43, 1993 (1991).

${ }^{53}$ M. Fuchs, M. Bockstedte, E. Pehlke, and M. Scheffler, Phys. Rev. B 57, 2134 (1998).

${ }^{54}$ L. Kleinman and D.M. Bylander, Phys. Rev. Lett. 48, 1425 (1982).

${ }^{55}$ X. Gonze, R. Stumpf, and M. Scheffler, Phys. Rev. B 44, 8503 (1991).

${ }^{56}$ S.G. Louie, S. Froyen, and M.L. Cohen, Phys. Rev. B 26, 1738 (1982).

${ }^{57}$ For our pseudopotentials we used as reference configurations and cutoff radii $\left(r_{s}^{\mathrm{cut}}, r_{p}^{\mathrm{cut}}, r_{d}^{\mathrm{cut}}, r_{f}^{\mathrm{cut}}\right)$ in atomic units: $\mathrm{N} 2 s^{2} 2 p^{3} 3 d^{0}$ $(1.5,1.5,1.5), \quad$ Al $3 s^{2} 3 p^{1} 3 d^{0} \quad(1.8,1.8,1.5), \quad$ Ga $4 s^{2} 4 p^{1} 4 d^{0}$ $(2.3,2.4,2.8), \quad$ Ga $\quad 3 d^{10} 4 s^{2} 4 p^{1} 4 f^{0} \quad(2.3,2.4,2.2,2.8), \quad$ In $5 s^{2} 5 p^{1} 5 d^{0}(2.3,2.4,2.8)$, In $4 d^{10} 5 s^{2} 5 p^{1} 5 g^{0}(2.3,2.4,2.2,2.8)$. In the case of nonlinear core-valence $\mathrm{XC}$, we used partial core densities with cutoff radii $1.3(\mathrm{Al}, \mathrm{Ga}), 1.8(\mathrm{In})$. The reference energies for the unbound unoccupied states were set to the $2 p$ $(\mathrm{N}), 3 p(\mathrm{Al})$ eigenvalues, and to $5 \mathrm{eV}(\mathrm{Ga} 4 d), 0 \mathrm{eV}(\mathrm{Ga} 4 f), 15$ $\mathrm{eV}(\mathrm{In} 5 d),-30 \mathrm{eV}$ (In $5 g$ ). For In we construct the $f$-like local potential from the In $5 g$ rather than the In $4 f$ state to achieve a smoother potential. 
${ }^{58}$ M. Städele, J.A. Majewski, and P. Vogl, Phys. Rev. B 56, 6911 (1997).

${ }^{59}$ M.B. Nardelli, K. Rapcewicz, and J. Bernholc, Phys. Rev. B 55, R7323 (1997).

${ }^{60}$ A. Dal Corso, A. Pasquarello, A. Baldereschi, and R. Car, Phys. Rev. B 53, 1180 (1996).

${ }^{61}$ D.R. Hamann, Phys. Rev. B 56, 14979 (1997).

${ }^{62}$ A. Trampert, O. Brandt, and K.H. Ploog, in Crystal Structure of Group III Nitrides, edited by J.I. Pankove and T.D. Moustakas, Semiconductors and Semimetals Vol. 50 (Academic, San Diego, 1998).

${ }^{63}$ M. Bernasconi, G.L. Chiarotti, and E. Tosatti, Phys. Rev. B 52, 9988 (1995).

${ }^{64}$ V.L. Moruzzi, J.F. Janak, and A.R. Williams, Calculated Electronic Properties of Metals (Pergamon, New York, 1978).

${ }^{65}$ The zero-point vibrational energy of the $\mathrm{N}_{2}$ dimer is $0.075 \mathrm{eV} /$ atom, by the frequency of the fundamental mode. For the crystals we estimate it as $0.10(\mathrm{AlN}), 0.06(\mathrm{GaN}), 0.04(\mathrm{InN}) \mathrm{eV} /$ atom, using a Debye model with the elastic constants of Ref. 79 as input. For the elemental metals Ref. 64 reports 0.04 (Al), 0.02 (Ga), and 0.01 (In) eV/atom.

${ }^{66}$ The fact that the formation enthalpies calculated within the pseudopotential framework slightly differ from the all-electron values (see Table I) does not affect our comparison of the LDA and the GGA.

${ }^{67}$ A. Kley, Ph.D. thesis, Technische Universität Berlin, 1997.

${ }^{68}$ S. Kurth, J.P. Perdew, and P. Blaha, Int. J. Quantum Chem. 75,
889 (1999).

${ }^{69}$ Following Ref. 70 an average value $\bar{s}$ of the scaled gradient may be defined through $F_{\mathrm{x}}(\bar{s})=E_{\mathrm{x}}^{\mathrm{GGA}}[n] / E_{\mathrm{x}}^{\mathrm{LDA}}[n]$.

${ }^{70}$ A. Zupan, K. Burke, M. Ernzerhof, and J.P. Perdew, J. Chem. Phys. 106, 10184 (1997).

${ }^{71}$ A.D. Becke, J. Chem. Phys. 104, 1040 (1996); 107, 8854 (1997); 109, 2092 (1998).

${ }^{72}$ K. Burke, M. Ernzerhof, and J.P. Perdew, Chem. Phys. Lett. 265, 115 (1997).

${ }^{73}$ T. Van Voorhis and G.E. Scuseria, J. Chem. Phys. 109, 400 (1998).

${ }^{74}$ J.P. Perdew, S. Kurth, A. Zupan, and P. Blaha, Phys. Rev. Lett. 82, 2544 (1999).

${ }^{75}$ CRC Handbook of Chemistry and Physics, 77th ed., edited by D.R. Lide (CRC Press, Boca Raton, FL, 1997).

${ }^{76}$ Semiconductors, edited by O. Madelung, Landolt-Börnstein, New Series, Group III, Vol. 17, Pts. a and b (Springer, Berlin, 1982).

${ }^{77}$ C. Kittel, Introduction to Solid State Physics (Wiley, New York, 1986).

${ }^{78}$ N.W. Ashcroft and N.D. Mermin, Solid State Physics (Saunders, Philadelphia, 1976).

${ }^{79}$ M.E. Sherwin and T.J. Drummond, J. Appl. Phys. 69, 8423 (1991).

${ }^{80}$ W.A. Harrison, Electronic Structure and the Properties of Solids (Dover, New York, 1989), p. 176.

${ }^{81}$ D.D. Wagman et al., J. Phys. Chem. Ref. Data 11 (Suppl. 2), 1 (1982). 